\title{
Staphylococcus aureus resistentes a meticilina en alimentos
}

\author{
Methicillin-resistant Staphylococcus aureus in food
}

Staphylococcus aureus resistente à meticilina nos alimentos

\author{
Silvia Monserrath Torres Segarra \\ storress@ucacue.edu.ec \\ https://orcid.org/0000-0002-4094-5522
}

\author{
Karla Estefanía Pacheco Cárdenas \\ karla.pacheco@ucacue.edu.ec \\ https://orcid.org/0000-0002-9571-9389
}

\author{
Maestría en Diagnóstico de Laboratorio Clínico y Molecular \\ de la Universidad Católica de Cuenca, Cuenca-Ecuador
}

Recibido 14 de mayo 2021 / Arbitrado y aceptado 08 de junio 2021 / Publicado 13 de diciembre 2021

\begin{abstract}
RESUMEN
Staphylococcus aureus es un microorganismo de importancia tanto a nivel hospitalario como en la comunidad; considerado parte de la microbiota normal en los humanos cuando existe condiciones apropiadas se comporta como oportunista, provoca infecciones leves hasta complicadas. Existen cepas de S. aureus multirresistentes a los antibióticos, debido a la adquisición por vía horizontal de genes de resistencia; entre ellas Staphylococcus aureus resistentes a meticilina (SARM), agentes etiológicos de infecciones graves relacionados directamente al consumo de alimentos contaminados. Objetivo. Analizar los posibles riesgos a los que se expone el ser humano al consumir alimentos contaminados por SARM, además de identificar los alimentos con mayor riesgo de contaminación y los factores que llevan a esta condición. Metodología. Mediante una revisión sistemática de estudios que indican la presencia de SARM en alimentos reportados en América latina. Las bases de datos consultadas: PubMed, SCOPUS, SCIELO y ProQuest mediante la declaración PRISMA. Se detectaron 30 estudios siendo elegibles 12 . Resultados. En América Latina se observó en Brasil mayor evidencia de SARM, luego Colombia y Chile; en los estudios encontrados indican que los alimentos con frecuencia mayor de contaminación de alimentos son los lácteos y sus derivados; productos cárnicos. Conclusiones. Se evidencia la estrecha relación entre el agente causal de contaminación que es SARM en alimentos a nivel de América Latina. El producto que más impacto ha presentado es la leche y sus derivados, los cuales al ser productos muy consumibles la salud de la población está en riesgo por la acción de enterotoxinas.
\end{abstract}

Palabras clave: Staphylococcus aureus; Beta-lactamasas; Meticilina; Alimentos; contaminación

\begin{abstract}
Staphylococcus aureus is an important microorganism both at hospital and community level; considered part of the normal microbiota in humans when appropriate conditions exist, it behaves as an opportunist, causing mild to complicated infections. There are strains of $\mathrm{S}$. aureus multiresistant to antibiotics, due to the horizontal acquisition of resistance genes, including methicillin-resistant Staphylococcus aureus (MRSA), etiological agents of serious infections directly related to the consumption of contaminated food. Objective. To analyze the possible risks to which humans are exposed when consuming food contaminated by MRSA, in addition to identifying the foods with the highest risk of contamination and the factors that lead to this condition. Methodology. Through a systematic review of studies indicating the presence of MRSA in food reported in Latin America. Databases consulted: PubMed, SCOPUS, SCIELO and ProQuest through the PRISMA statement. Thirty studies were detected and 12 were eligible. Results. In Latin America, the greatest evidence of MRSA was observed in Brazil, followed by Colombia and Chile; the studies found indicate that the foods with the highest frequency of food contamination are dairy products and their derivatives; meat products. Conclusions. There is evidence of a close relationship between the causal agent of MRSA contamination in food in Latin America. The product that has had the greatest impact is milk and its derivatives, which, being highly consumable products, put the health of the population at risk due to the action of enterotoxins.
\end{abstract}

Key words: Staphylococcus aureus, beta-lactamases, methicillin, food, contamination 


\section{RESUMO}

Staphylococcus aureus é um microorganismo importante tanto a nível hospitalar como comunitário; considerado parte da microbiota normal em humanos quando existem condições apropriadas, comporta-se como oportunista, causando infecções leves a complicadas. Existem estirpes de $S$. aureus multiresistentes aos antibióticos, devido à aquisição horizontal de genes de resistência, incluindo Staphylococcus aureus resistente à meticilina (MRSA), agentes etiológicos de infecções graves directamente relacionadas com o consumo de alimentos contaminados. Objectivo. Analisar os possíveis riscos a que os seres humanos estão expostos ao consumir alimentos contaminados por MRSA, para além de identificar os alimentos com maior risco de contaminação e os factores que levam a esta condição. Metodologia. Através de uma revisão sistemática de estudos que indicam a presença de MRSA nos alimentos reportados na América Latina. Bases de dados consultadas: PubMed, SCOPUS, SCIELO e ProQuest, utilizando a declaração PRISMA. Foram detectados trinta estudos, 12 dos quais eram elegíveis. Resultados. Na América Latina, a maior evidência de MRSA foi observada no Brasil, seguido pela Colômbia e Chile; os estudos encontrados indicam que os alimentos com maior frequência de contaminação de alimentos são produtos lácteos e seus derivados; produtos de carne. Conclusões. Há provas de uma relação estreita entre o agente causal da contaminação por MRSA nos alimentos na América Latina. $O$ produto que tem tido maior impacto é o leite e seus derivados, que como são produtos altamente consumíveis, a saúde da população está em risco devido à acção das enterotoxinas.

Palavras-chave: Staphylococcus aureus; betalactamases; meticilina; alimentos; contaminação

\section{INTRODUCCIÓN}

El Staphylococcus aureus (S. aureus), es uno de los patógenos que más afección ha generado a los seres humanos fue descubierto en el año 1880 observada por vez primera por Alexander Ogston. Dicho microorganismo fue aislado a partir de una infección de herida quirúrgica, en donde se observó que era capaz de generar abscesos, hecho que se pudo notar cuando se inoculó a cobayos y ratones. Posteriormente, en 1882 , se acuñó el término
Estafilococo para referirse al género y luego en el año 1884 Rosenbach dividió el género en las especies $S$. aureus y S. albus; poco tiempo después, se incorporó la especie S. epidermis, en base a los resultados obtenidos por Cowan al emplear la prueba de la coagulasa $(1,2)$.

El S. aureus forma parte de la microbiota normal de la piel y mucosas, en especial la nasal, encontrándose entre el 20 - 30\% de los individuos. Si su hábitat es alterado, se comporta como un patógeno oportunista. Esto se debe a que $S$. aureus posee un conjunto de factores de virulencia y la capacidad de adquirir resistencia a la mayoría de los antibióticos. De hecho, la aparición de nuevos clones multi-resistentes es cada vez más frecuente, no solo a nivel intrahospitalario, sino a nivel comunitario (1-4).

Las cepas de Staphylococcus aureus resistente a la meticilina (SARM), son consideradas como patógenos de relevancia epidemiológica y clínica, debido a su estrecha relación con las Infecciones Asociadas a la Asistencia Sanitaria (IAAS). Es así, que este patógeno forma parte del grupo de microorganismo que generan graves enfermedades nosocomiales, debido en gran medida a la resistencia generada a la meticilina e incluso a toda la familia de los -lactámicos (4).

La meticilina es un antibiótico sintético que pertenece al grupo de los -lactámicos que se introdujo como un medicamento de elección para combatir las cepas de $S$. aureus resistentes a la penicilina, fenómeno asociado a la producción de una enzima -lactamasa 
codificada por el gen blaZ. (5). Los estafilococos presentan el cromosoma mec y en especial el gen mecA es el responsable de la resistencia a los $\beta$-lactámicos característica importante para la clasificación como SARM (6).

En la actualidad se establece que las cepas SARM no solamente son causantes de infecciones nosocomiales, sino que también se asocian a infecciones comunitarias. Las cepas SARM son capaces de colonizar órganos y tejidos en los animales de granja. En consecuencia pueden entrar en contacto con el ser humano ya sea de forma directa o indirecta a partir de sus derivados alimenticios (7).

La presencia de S. aureus en alimentos representa un problema latente, es así, que es un microorganismo indicador de contaminación, y la presencia de este se relaciona con la mala manipulación por parte de las personas que están en contacto directo con el proceso de la cadena productiva. Es decir, la falta de aplicación de buenas prácticas de manufactura especialmente en alimentos de consumo fresco como es el caso de los cárnicos, de los lácteos y sus derivados. Otro punto interesante resulta los lugares en donde se generan los productos, éstos espacios deben cumplir con protocolos y normativas que garanticen una adecuada producción (8).

Elconocimientodeestasformasdeinfección es muy relevante para los profesionales de la salud que se ven involucrados en el control de estas infecciones o en las investigaciones que se llevan a cabo sobre nuevas formas de contagio de las cepas SARM. Entre otras cosas, este conocimiento permitiría relacionar consecuencias a futuro las cuales se deriven de la ingesta de dichos alimentos contaminados. Por ello se busca dar respuesta a las siguientes interrogantes: ¿Pueden estas cepas SARM considerarse únicamente como causantes de infecciones zoonóticas? Si no es así, ¿Pueden en algún momento pasar al hombre por medio del consumo de los alimentos contaminados? ¿Es importante comunicar a las entidades de salud sobre los alimentos que presenten SARM y que sean de consumo frecuente en el país?

Existen diversas publicaciones relacionadas con el problema de salud que involucran la presencia de SARM como, las infecciones asociadas y la diversidad de los reservorios, generando mayor importancia por la elevada prevalencia en los animales de granja prioritariamente en caballos, pollos y vacas (4).

Con respecto a las enfermedades transmitidas por alimentos (ETA), se relaciona con las infecciones causadas por S. aureus, productores de enterotoxina estafilococócica, la cual genera sintomatología de intoxicación típica de un proceso gastroentérico, relacionada íntimamente con la incorrecta manipulación de los diferentes alimentos por parte del personal (9). Existe relación de la contaminación con SARM en alimentos, ya que se inicia desde la calidad de vida de los animales de ordeño, en especial cuando padecen de mastitis por lo que se considera como punto de inicio la contaminación de los productos lácteos; sumado la incorrecta aplicación de las Buenas Prácticas de Manufactura tanto en 
los procesos de obtención de la materia prima como en el personal que trabaja dentro de la planta procesadora (8).

Por ello el objetivo de este estudio es analizar los posibles riesgos a los que se expone el ser humano al consumir alimentos contaminados por SARM, además de identificar los alimentos con mayor riesgo de contaminación y los factores que llevan a esta condición.

\section{MATERIALES Y MÉTODOS}

Se realizó una revisión sistemática de estudios asociados a la presencia de Staphylococcus aureus resistentes a la meticilina en alimentos reportados en América latina. Para la clasificación de las fuentes consultadas se empleó la declaración PRISMA, la búsqueda bibliográfica se efectuó en las bases de datos electrónicas PubMed, SCOPUS, SCIELO y ProQuest con los siguientes términos descriptores: Staphylococcus aureus, SARM, MRSA, Foodborne Diseases, Food Microbiology.

El periodo de recogida de artículos fue de 2000 al 2020 en los idiomas inglés, español y portugués. Para el desarrollo del presente estudio fueron consultados 30 estudios, de los cuales se eligieron $12 / 30$. Fueron excluidos una totalidad de 18 estudios debido a duplicidad, en caso de tratarse de estudios de meta-análisis o en su defecto estudios fuera de América Latina, ver Figura 1.

Criterios de inclusión. Artículos que se relacionen con la parte genética del Staphylococcus aureus resistentes a la meticilina. Documentos que indiquen métodos de resistencia fenotípica. Artículos que impliquen la presencia de Staphylococcus aureus resistente a la meticilina en alimentos.

Criterios de exclusión: Artículos científicos que no estén relacionados con el tema, o que no estén publicados en revistas de alto impacto, artículos que se repitan en las bases de datos. Documentos que no estén dentro del periodo considerado para la revisión.

Estrategia de búsqueda. Los términos de búsqueda que se emplearon fueron las siguientes palabras clave: Staphylococcus aureus, SARM, MRSA, "Methicillin-Resistant Staphylococcus aureus", "Foodborne diseases" "Food Microbiology"; "Chicken meat”, "Chickens”, "Turkey meat", "Food Contamination”, "Seafood”, cárnicos, derivados lácteos, intoxicación alimentaria, patogenicidad, virulencia, "South america", "Latin America"; además, del empleo de los operadores booleanos "AND", "OR" y "NOT". 

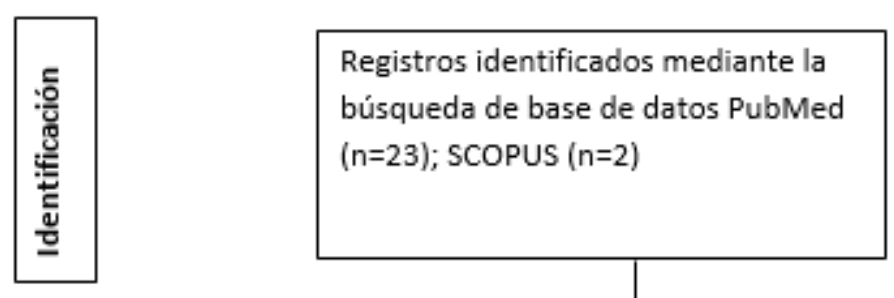

Registros adicionales identificados a través de otras fuentes SCIELO ( $n=3)$; ProQuest $(n=2)$
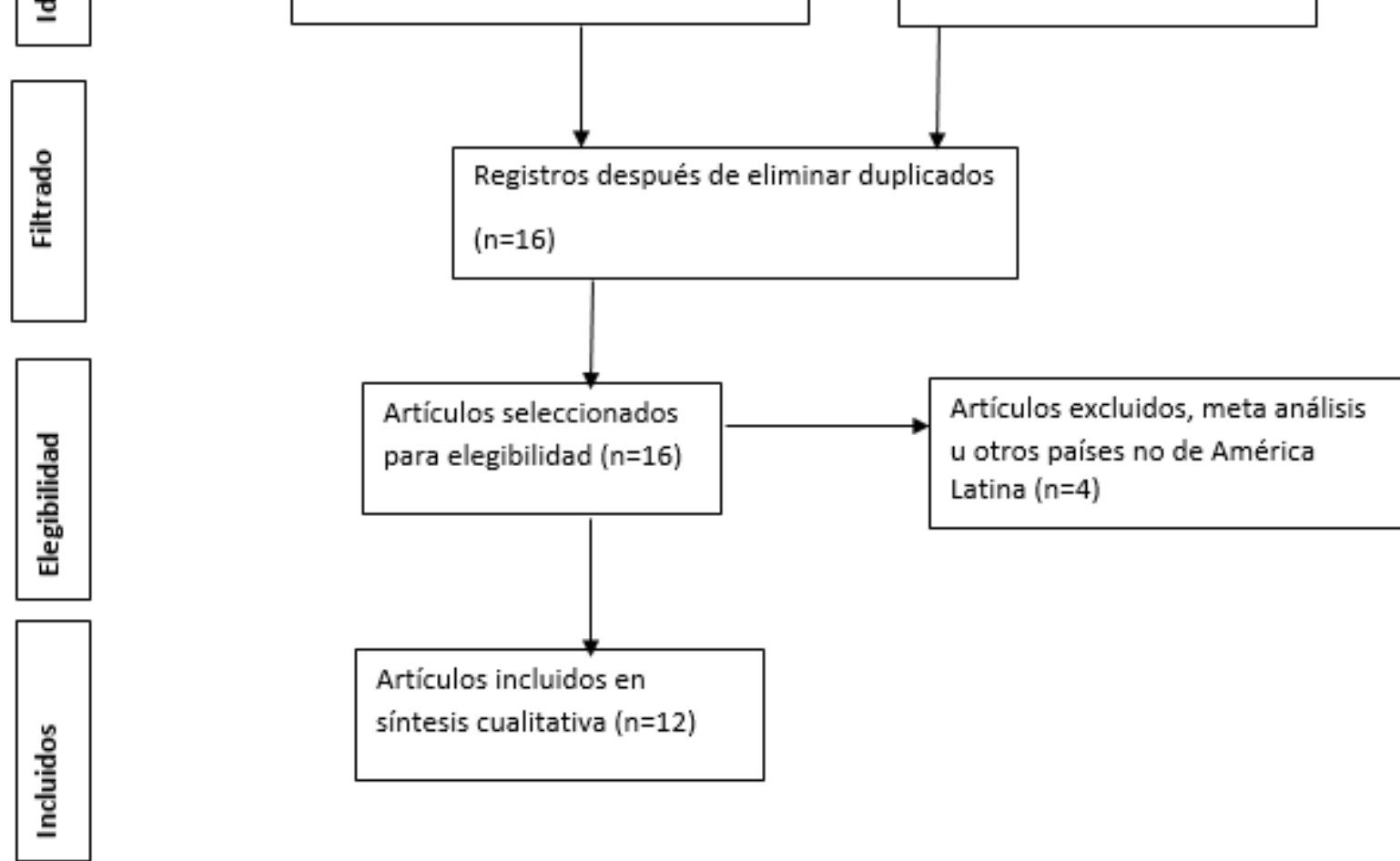

Figura 1. Flujograma de elegibilidad de los estudios relacionados con Staphylococcus aureus resistentes a la meticilina en alimentos reportados en América latina.

\section{DESARROLLO Y DISCUSIÓN}

El S. aureus constituye un agente etiológico frecuente de intoxicaciones alimentarias y se encuentra asociado a diversos alimentos. Las ETA se encuentran ampliamente extendidas y constituyen un problema de salud pública, tanto en países desarrollados como en países en vías de desarrollo. SARM considerado como un patógeno oportunista; causa varias infecciones tanto en humanos como en animales y una de sus características es la resistencia a los $\beta$-lactámicos.

En la Tabla 1, se visualiza los estudios a nivel de América Latina, en donde se refleja la importancia del periodo de tiempo instaurado para la presente investigación que se contrasta con la gran cantidad y variedad de alimentos contaminados con SARM. 
Tabla 1. Artículos elegibles con el año de publicación e indicadores higiénicos sanitarios.

\begin{tabular}{ccc}
\hline País & Año & Indicadores higiénicos sanitarios \\
\hline Brasil & 2011 & Queso \\
& & Bento \\
Brasil & 2011 & Sushi \\
Brasil & 2014 & Leche de búfalo fresca \\
Brasil & 2015 & Leche y productos lácteos \\
Brasil & 2015 & Carne cruda \\
Colombia & 2015 & Alimentos varios preparados \\
Colombia & Hamburguesas crudas \\
Chile & 2016 & Sánduches \\
Brasil & 2018 & Queso doble crema \\
Brasil & 2019 & Queso \\
Brasil & 2019 & Carne de cerdo \\
Colombia & 2020 & Tilapia \\
\hline
\end{tabular}

La Figura 2 permite identificar que el seguido de Colombia con 3 artículos 23.07\% país que cuenta con mayor cantidad de y finalmente Chile con un solo artículo que publicaciones asociadas a la temática es Brasil corresponde al 7.7\%. con 8 artículos que corresponde al 69.23\%,

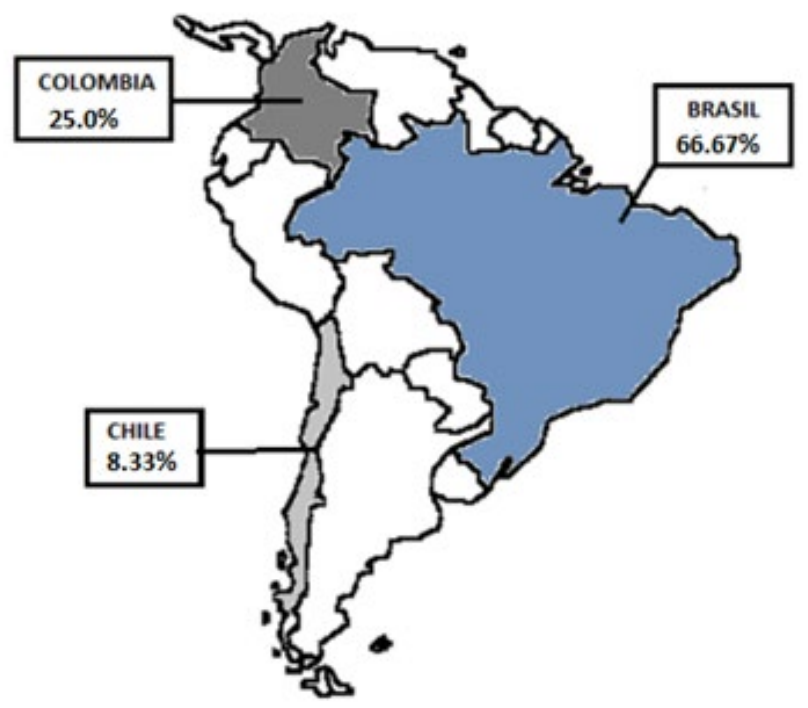

Figura 2. Distribución en porcentaje de acuerdo con los artículos encontrados a nivel de América Latina. 
En la Tabla 2 se observa la elevada frente a todos los $\beta$-lactámicos, empleados prevalencia del gen mecA, importante comúnmente en la profilaxis clínica, lo cual indicador paralaidentificación deSARM, elcual genera inconvenientes en el área de salud. está asociado a la resistencia antimicrobiana

Tabla 2. Datos identificados de los indicadores higiénicos sanitarios de alimentos de las fuentes consultadas.

\begin{tabular}{|c|c|c|c|c|}
\hline País & Alimento & $\begin{array}{l}\text { Gen y o enzima } \\
\text { identificada }\end{array}$ & $\begin{array}{l}\text { Prevalencia en el } \\
\text { producto }\end{array}$ & Dato de interés \\
\hline Brasil & $\begin{array}{l}\text { Queso } \\
\text { Bento } \\
\text { Sushi }\end{array}$ & mecA & $\begin{array}{l}\text { Queso } 1 \% \\
\text { Bento 1\% } \\
\text { Sushi 3\% }\end{array}$ & $\begin{array}{l}\text { Gen mecA inactivo debido a la } \\
\text { presencia de genes reguladores. } \\
\text { Se considera a los alimentos como } \\
\text { portadores del gen mecA. }\end{array}$ \\
\hline Brasil & $\begin{array}{l}\text { Leche de búfalo } \\
\text { fresca }\end{array}$ & $\beta$-lactamasa & $\begin{array}{l}83,0 \% \text { de las cepas } \\
\text { aisladas de S. aureus }\end{array}$ & $\begin{array}{l}\text { Son productoras de } \\
\beta \text {-lactamasa, las cepas } \\
\text { estudiadas, además se evaluaron } \\
\text { la presencia de enterotoxinas }\end{array}$ \\
\hline Brasil & $\begin{array}{l}\text { Leche y productos } \\
\text { lácteos }\end{array}$ & $\mathrm{N} / \mathrm{A}$ & $\begin{array}{l}93,6 \% \text { de las cepas de } \\
\text { S. aureus albergaban } \\
\text { perfiles variables de } \\
\text { genes de enterotoxina } \\
\text { estafilocócica }\end{array}$ & $\begin{array}{l}\text { Riesgo alto de contaminación } \\
\text { alimentaria }\end{array}$ \\
\hline Brasil & $\begin{array}{l}\text { Carne cruda. } \\
\text { Alimentos varios } \\
\text { preparada }\end{array}$ & mecA & $\begin{array}{l}\text { Carne cruda } \mathbf{2 8 . 1 \%} \\
\text { Carne de vacuno } 23.3 \% \\
\text { Pollo } 23.3 \% \\
\text { Cerdo } 37.5 \% \\
\text { Pescado } 30.0 \% \\
\text { Alimentos varios } \\
\text { preparados } \mathbf{9 . 5 \%} \\
\text { Con pollo } 5.6 \% \\
\text { Con cerdo } 6.7 \% \\
\text { Con pescado } 22.2 \%\end{array}$ & $\begin{array}{l}\text { De un total de } 114 \text { muestras } \\
\text { tomadas de comida para ser } \\
\text { preparada y distribuida en un } \\
\text { hospital público; las } 98 \text { dieron } \\
\text { como positivos para SARM }\end{array}$ \\
\hline Brasil & $\begin{array}{l}\text { Hamburguesas } \\
\text { crudas } \\
\text { Sánduches }\end{array}$ & $\mathrm{N} / \mathrm{A}$ & $\begin{array}{l}\text { Hamburguesas 32\% } \\
\text { Sánduches } 8 \%\end{array}$ & $\begin{array}{l}\text { Identificación mediante pruebas } \\
\text { de sensibilidad a los antibióticos }\end{array}$ \\
\hline Colombia & $\begin{array}{l}\text { Queso doble } \\
\text { crema }\end{array}$ & mecA & $\begin{array}{l}\text { Se detectó el } 18.2 \% \\
\text { de cepas S. aureus } \\
\text { identificadas, } \\
\text { correspondiendo a una } \\
\text { prevalencia del } 9.0 \% \text { en } \\
\text { las muestras analizadas }\end{array}$ & $\begin{array}{l}\text { Identificación mediante prueba } \\
\text { PCR }\end{array}$ \\
\hline
\end{tabular}




\begin{tabular}{|c|c|c|c|c|}
\hline País & Alimento & $\begin{array}{l}\text { Gen y o enzima } \\
\text { identificada }\end{array}$ & Prevalencia en el producto & Dato de interés \\
\hline Colombia & Queso & mecA & $\begin{array}{l}\text { Se caracterizaron } 65 \\
\text { aislados de estafilococos } \\
\text { coagulasa-positivos y el } \\
18,5 \% \text { de ellos eran SARM }\end{array}$ & $\begin{array}{l}\text { Identificación mediante } \\
\text { prueba PCR y sensibilidad a } \\
\text { los antibióticos }\end{array}$ \\
\hline Chile & Carne de cerdo & mecA & $\begin{array}{l}\text { carne de cerdo } 33,9 \% \text {; } \\
\text { carne al por menor no } \\
\text { envasada } 43,1 \% \text {; } \\
\text { carne al por menor } \\
\text { envasada } 5,3 \%\end{array}$ & $\begin{array}{l}\text { Identificación mediante } \\
\text { prueba PCR y pruebas } \\
\text { bioquímicas }\end{array}$ \\
\hline Brasil & Tilapia & mecA & $\begin{array}{l}\text { De } 100 \text { muestras las } 12 \\
\text { resultan positivas }\end{array}$ & $\begin{array}{l}\text { Identificación mediante } \\
\text { prueba PCR }\end{array}$ \\
\hline Brasil & Queso de cabra & $\beta$-lactamasa & $\begin{array}{l}\text { El } 7.4 \% \text { es decir } 4 \text { de } 54 \\
\text { muestras eran resistentes } \\
\text { a la oxacilina por lo tanto } \\
\text { también resistente a } \\
\text { meticilina }\end{array}$ & $\begin{array}{l}\text { No se detectó el gen mecA, } \\
\text { pero si la producción de } \\
\beta \text {-lactamasa }\end{array}$ \\
\hline Brasil & Queso artesanal & mecA & $\begin{array}{l}\text { Se detectaron } 16 \text { aislados } \\
\text { que contenían el gen mecA } \\
\text { en muestras de } \\
\text { queso no pasteurizado y de } \\
\text { manipuladores de queso. }\end{array}$ & $\begin{array}{l}\text { Identificación por PCR } \\
\text { multiplex para confirmar la } \\
\text { presencia de S. aureus }\end{array}$ \\
\hline Colombia & Leche & mecA & $\begin{array}{l}\text { El } 27 \% \text { de las cepas } \\
\text { evaluadas se consideran } \\
\text { como SARM }\end{array}$ & $\begin{array}{l}\text { Presencia de gen blaZ, el cual } \\
\text { es productor de } \beta \text {-lactamasa }\end{array}$ \\
\hline
\end{tabular}

No aplica se considera como N/A; debido a que se trata de otra consideración para identificar a SARM.

En función a la revisión se evidencia que, a nivel de América Latina el país que más estudios ha realizado con respecto a SARM en los últimos años es Brasil, seguido de Colombia y Chile; en todos ellos se refleja que los alimentos con mayor contaminación son los lácteos y productos derivados como los quesos y la leche de ganado bovino.

Se evidencia que los alimentos con mayor grado de contaminación por $\mathrm{S}$. aureus son la leche y sus derivados seguido de productos cárnicos, sin embargo, la importancia radica en la amplia variedad de cepas productoras de resistencia a los antimicrobianos.

\section{Discusión}

Staphylococcus aureus, es un patógeno que se encuentra fácilmente como agente causal de mastitis clínicas crónicas o recurrentes, lo que se concuerda con Guimaraes, F. et al. (10) quienes relacionan que obtener productos derivados de animales enfermos conlleva un 
alto riesgo de contraer enfermedad, además se indica que son los responsables de generar enterotoxinas pirogénicas, las mismas que tienen la capacidad de generar el síndrome de choque tóxico las cuales están relacionadas con la intoxicación alimentaria $(11,12)$, así como lo menciona Erdem, S. et al (13) en su investigación indica que identificó el gen que causa la producción de las enterotoxinas teniendo una frecuencia del $41.6 \%$ de las muestras analizadas.

El análisis de leche de búfalo, bajo normas de asepsia se tomaron muestras donde indican que $83 \%$ de las cepas de $S$. aureus presentaron resistencia ya que se han identificado como productoras de $\beta$-lactamasa $(11,14)$. Además, en el análisis de quesos de cabra obtienen como resultado la hiperproducción de ésta enzima la cual está relacionada con el gen blaZ, sin embargo no se evidencia la presencia del gen mecA $(15,16)$. relacionando con el estudio realizado por Alves, $M$. et al (17) en donde han analizado muestras de leche de animales con mastitis en donde no se logra evidenciar el gen mecA; pero en este estudio se investigó el perfil genotípico y clonal de SARM.

De la investigación realizada, se observó que existe contaminación alta a nivel de la elaboración de diferentes tipos de quesos como es el caso del artesanal donde se encontró la presenciad del gen mecA $(18,19)$ mediante el procedimiento de PCR indicando que se trata de SARM (20). En un estudio realizado por Herrera, FC et al, que determinaron la presencia de estafilococo coagulasa positivos en un $18.3 \%$ de las muestras. Lo relevante del estudio es que indica una contaminación donde se sugiere que la fuente de ello es la humana a través de alimentos los cuales pueden contribuir a la diseminación mundial de clones SAMR adquiridos en la comunidad (21); lo mismo indican en el análisis realizado por Matyi, S. et al (22), en el cual mediante técnica PCR lograron identificar dos cepas de S. aureus, identificando el gen mecA el cual involucra la resistencia a la meticilina.

La contaminación por S. aureus se evidencia también en cárnicos; como indica los resultados generados en un año de investigación donde el $1.9 \%$ correspondía positivos para SARM, este estudio fue realizado por $\mathrm{Ge}$, B et al., detectando en carnes de venta al por menor (23); lo que relaciona con los artículos encontrados para la presente revisión donde en carne cruda se relaciona un $28.1 \%$ de vacuno, pollo un $23.3 \%$; cerdo $37.5 \%$ y pescado un $30.0 \%$ (24). Además, se encontró otro estudio en el cual se encuentra tilapia contaminada con SARM, relacionando con los utensilios de cocina utilizados para el expendio del producto $(19,25)$.

Costa et al., (24) encontró en varios alimentos preparados con los siguientes porcentajes $9.5 \%$ que se divide en alimentos con pollo como ingrediente en un $5.6 \%$, con cerdo un $6.7 \%$ y con pescado un $22.2 \%$; lo que se concuerda con la investigación en alimentos de comida rápida como hamburguesas y sánduches en donde se encontró la presencia de SARM (15). De igual manera, en un estudio realizado en lowa se evidencia la frecuencia del aislamiento de SARM en general fue del $15.7 \%$ indicando que los cárnicos están 
susceptibles a contaminación; para la carne de res indica el $100 \%$, pavo $77.8 \%$, pollo el $71.4 \%$ y el $58.3 \%$ para la carne de cerdo (26).

Se ha identificado la presencia de SARM en carne de cerdo, pero no se demostró la presencia del gen mecA este estudio se realizó en Chile (27). Sin embargo, Buyukcangaz E. et al., (28) en un estudio realizado indica que se detectó el gen mecA únicamente en la carne de cerdo pero lo que representa una baja prevalencia, ambos estudios utilizaron la técnica de PCR para la identificación específica del gen.

S.aureus, es un patógeno que coloniza la zona de la nasofaringe, mucosas y piel lo que relaciona de manera directa que los alimentos se contaminan al existir la falta de aplicación de las BPM por parte de los manipuladores, materia prima o en utensilios para la preparación y distribución de productos $(8,9,29)$; lo que se confirma con los estudios realizados por Jiménez et al., (30) en donde se investigaron a manipuladores de pescado encontrando que en el $3 \%$ de portadores asintomáticos presentó SARM; determinando que la transmisión puede darse por contacto directo de las personas con los productos alimenticios, siempre que no se cumpla con vigilancia sanitaria (30).

Las ETA suceden a nivel mundial afectando a una gran cantidad de población lo que resulta preocupante para las entidades de control sanitario, debido a que se estima que miles de personas llegan a hospitalizarse por este tipo de afección llevando incluso a un diagnóstico erróneo (31). Lo que relaciona con el uso indiscriminado de antibióticos cuando los animales tienen infección (21,32). Lo que se refuerza con lo expresado en la investigación realizada por Zendeja et al., (33) donde indica que las enfermedades infecciosas son causadas por la ingesta de toxinas de $S$. aureus los cuales causan un impacto grande en los individuos que la padecen.

En el transcurso del proceso de elaboración de la presente revisión sistemática, se encontró con algunos inconvenientes en cuanto a la falta de información a nivel de América Latina, lo que a su vez evidencia la falta de investigación en cuanto a la contaminación de alimentos por SARM, lo que de cierta manera es un llamado a los investigadores a desarrollar mayor interés en cuanto al tema.

Como sugerencia para futuras investigaciones se propone ampliar el rango de estudio considerando a todo el continente americano, para de esta manera ampliar la recopilación de artículos publicados y con ello la comparación referente a la contaminación de alimentos por S. aureus.

\section{CONCLUSIÓN}

Se evidenció la estrecha relación entre los agentes causales de contaminación que en este caso es $S$. aureus resistentes a la meticilina en alimentos a nivel de América Latina, a pesar de no contar con información amplia que aborde la temática, se reflejó, que la presencia de SARM se incrementa debido a la manipulación inadecuada de la materia prima o durante su proceso de elaboración. 
El alimento que más impacto ha presentado es la leche y productos derivados, mismos que al ser uno de los productos de mayor consumo pueden poner en riesgo la salud de la población e incrementando el impacto a nivel de la salud pública, debido a la acción de enterotoxinas las cuales han causado procesos gastroentéricos agudos.

\section{REFERENCIAS BIBLIOGRÁFICAS}

1. Harkins $C P$, Pichon $B$, Doumith $M$, Parkhill J, Westh H, Tomasz A, et al. Methicillinresistant Staphylococcus aureus emerged long before the introduction of methicillin into clinical practice. Genome Biol. 20 de julio de 2017;18(1):130.

2. Pasachova Garzón J, Ramírez Martínez S, Muñoz Molina L, Pasachova Garzón J, Ramírez Martínez S, Muñoz Molina L. Staphylococcus aureus: generalidades, mecanismos de patogenicidad y colonización celular. Nova. diciembre de 2019;17(32):25-38.

3. Bastidas CA, Villacrés-Granda I, Navarrete D, Monsalve M, Coral-Almeida M, Cifuentes SG. Antibiotic susceptibility profile and prevalence of mecA and lukS-PV/lukF-PV genes in Staphylococcus aureus isolated from nasal and pharyngeal sources of medical students in Ecuador [Internet]. Vol. 12, Infection and Drug Resistance. Dove Press; 2019 [citado 21 de mayo de 2020]. p. 2553-60. Disponible en: https:// www.dovepress.com/antibiotic-susceptibilityprofile-and-prevalence-of-meca-and-lukspvlu-peer-reviewed-article-IDR

4. Kale $P$, Dhawan $B$. The changing face of community-acquired methicillin-resistant Staphylococcus aureus. Indian J Med Microbiol. septiembre de 2016;34(3):275-85.

5. Rodvold KA, McConeghy KW. Methicillinresistant Staphylococcus aureus therapy: past, present, and future. Clin Infect Dis Off Publ Infect Dis Soc Am. enero de 2014;58 Suppl 1:S20-27.

6. Lakhundi S, Zhang K. MethicillinResistant Staphylococcus aureus: Molecular Characterization, Evolution, and Epidemiology. Clin Microbiol Rev [Internet]. 1 de octubre de 2018 [citado 22 de mayo de 2020];31(4). Disponible en: https://cmr.asm.org/ content/31/4/e00020-18

7. Aires-de-Sousa M. Methicillin-resistant Staphylococcus aureus among animals: current overview. Clin Microbiol Infect. 1 de junio de 2017;23(6):373-80.

8. Figueroa $G G$, Navarrete $W P$, Caro $C M$, Troncoso H M, Faúndez $Z$ G. Portación de Staphylococcus aureus enterotoxigénicos en manipuladores de alimentos. Rev Médica Chile. agosto de 2002;130(8):859-64.

9. Peña YP, Hernández ME, Castillo VL, López NA, Muñoz YP, Rodríguez PS. Resistencia antimicrobiana en cepas de estafilococos coagulasa positiva aisladas en alimentos y manipuladores. Rev Cuba Aliment Nutr. 28 de diciembre de 2015;25(2):245-60.

10. Guimarães FF, Manzi MP, Joaquim SF, Richini-Pereira VB, Langoni $H$. Short communication: Outbreak of methicillinresistant Staphylococcus aureus (MRSA)associated mastitis in a closed dairy herd. J Dairy Sci. 1 de enero de 2017;100(1):726-30.

11. Oliveira AAF, Pinheiro JW, Mota RA, Cunha MLRS, Lopes CAM, Rocha NS. Phenotype characterization of Staphylococcus species strains isolated from buffalo (Bubalus bubalis) milk. J Vet Diagn Invest. 1 de noviembre de 2011;23(6):1208-11.

12. Silveira-Filho VM, Luz IS, Campos APF, Silva WM, Barros MPS, Medeiros ES, et al. Antibiotic Resistance and Molecular Analysis of Staphylococcus aureus Isolated from Cow's Milk and Dairy Products in Northeast Brazil. J Food Prot. 1 de abril de 2014;77(4):583-91. 
13. Saka E, Terzi Gulel G. Detection of Enterotoxin Genes and Methicillin-Resistance in Staphylococcus aureus Isolated from Water Buffalo Milk and Dairy Products: Detection of enterotoxin genes.... J Food Sci. junio de 2018;83(6):1716-22.

14. Castellano González MJ, Perozo-Mena AJ. Mecanismos de resistencia a antibióticos $\beta$-lactámicos en Staphylococcus aureus. Kasmera. junio de 2010;38(1):18-35.

15. Haskell KJ, Schriever SR, Fonoimoana KD, Haws B, Hair BB, Wienclaw TM, et al. Antibiotic resistance is lower in Staphylococcus aureus isolated from antibiotic-free raw meat as compared to conventional raw meat. PLoS ONE [Internet]. 10 de diciembre de 2018 [citado 9 de junio de 2021];13(12). Disponible en: https://www.ncbi.nlm.nih.gov/pmc/articles/ PMC6287829/

16. Short communication: High frequency of $\beta$-lactam-resistant Staphylococcus aureus in artisanal coalho cheese made from goat milk produced in northeastern Brazil - Journal of Dairy Science [Internet]. [citado 26 de febrero de 2021]. Disponible en: https://www. journalofdairyscience.org/article/S00220302(19)30494-1/fulltext

17. Alves $M$ de FNF, Penna $B$, Pereira RFA, Geraldo RB, Folly E, Castro HC, et al. First report of meticillin-resistant Staphylococcus aureus harboring mecC gene in milk samples from cows with mastitis in southeastern Brazil. Braz J Microbiol. diciembre de 2020;51(4):2175-9.

18. Freitas Ribeiro L, Akira Sato R, de Souza Pollo A, Marques Rossi GA, do Amaral LA. Occurrence of Methicillin-Resistant Staphylococcus spp. on Brazilian Dairy Farms that Produce Unpasteurized Cheese. Toxins. 8 de diciembre de 2020;12(12):779.

19. Federal University of Sãn Francisco Valley (UNIVASF), Pernambuco, Brazil, Correia TMA, Oliveira APD, Miyasato IF, Santos TMB, Dias
FS. Characterization of Staphylococcus aureus isolated from tilapia and utensils used in the commerciali- zation of tilapia in the street markets of a semi-arid Brazilian municipality [pdf]. Acta Sci Pol Technol Aliment. 30 de diciembre de 2019;18(4):413-25.

20. Herrera A. F, Santos B. J. PRESENCIA DE Staphylococcus aureus METICILINARESISTENTES EN QUESO DOBLE CREMA ARTESANAL. Rev UDCA Actual Amp Divulg Científica. junio de 2015;18(1):29-37.

21. Herrera FC, García-López M-L, Santos JA. Short communication: Characterization of methicillin-resistant Staphylococcus aureus isolated from raw milk fresh cheese in Colombia. J Dairy Sci. 1 de octubre de 2016;99(10):7872-6.

22. Matyi SA, Dupre JM, Johnson WL, Hoyt PR, White DG, Brody $T$, et al. Isolation and characterization of Staphylococcus aureus strains from a Paso del Norte dairy. J Dairy Sci. junio de 2013;96(6):3535-42.

23. Ge B, Mukherjee $S$, Hsu C-H, Davis JA, Tran TTT, Yang $Q$, et al. MRSA and multidrugresistant Staphylococcus aureus in U.S. retail meats, 2010-2011. Food Microbiol. abril de 2017;62:289-97.

24. Costa WLR, Ferreira J dos S, Carvalho JS, Cerqueira ES, Oliveira LC, Almeida RC de C. Methicillin-Resistant Staphylococcus aureus in Raw Meats and Prepared Foods in Public Hospitals in Salvador, Bahia, Brazil. J Food Sci. 2015;80(1):M147-50.

25. Jordá GB, Marucci RS, Guida AM, Pires PS, Manfredi EA. Portación y caracterización de Staphylococcus aureus en manipuladores de alimentos. Rev Argent Microbiol. 2012;5.

26. PubMed Central Full Text PDF [Internet]. [citado 9 de junio de 2021]. Disponible en: https://www.ncbi.nlm.nih.gov/pmc/articles/ PMC6287829/pdf/pone.0206712.pdf

27. Prevalence and Characterization of 
Staphylococcus aureus Strains in the Pork Chain Supply in Chile | Foodborne Pathogens and Disease [Internet]. [citado26 defebrero de2021]. Disponible en: https://www.liebertpub.com/ doi/10.1089/fpd.2017.2381?url_ver=Z39.882003\&rfr_id=ori\%3Arid\%3Acrossref.org\&rfr_ dat $=c r \_p u b++0$ pubmed\&

28. Buyukcangaz E, Velasco V, Sherwood JS, Stepan RM, Koslofsky RJ, Logue CM. Molecular Typing of Staphylococcus aureus and Methicillin-Resistant S. aureus (MRSA) Isolated from Animals and Retail Meat in North Dakota, United States. Foodborne Pathog Dis. julio de 2013;10(7):608-17.

29. Salina $M$, Scholz $L$, Servián $N$, Romero $M$, Samudio T, Ruiz V, et al. Staphylococcus Aureus in food manipulators of gastronomic services of Asunción, Paraguay (2017). Rev Salud Publica Parag. 30 de diciembre de 2018;8(2):28-33.

30. Jiménez JT, Mata YCO, Díaz DIO, Damián LL, Salgado JP, Forero AF, et al. Portadores asintomáticos de Staphylococcus aureus meticilino resistentes (MRSA) en pescadores y horticultores de Guerrero, México. J Negat No Posit Results. 1 de noviembre de 2020;5(12):1482-9.
31. da Silva AC, Rodrigues MX, Silva NCC. Methicillin-resistant Staphylococcus aureus in food and the prevalence in Brazil: a review. Braz J Microbiol. 1 de marzo de 2020;51(1):34756.

32. Jiménez Velásquez $\mathrm{S}$ del $\mathrm{C}$, Torres Higuera LD, Parra Arango JL, Rodríguez Bautista JL, García Castro FE, Patiño Burbano RE. Perfil de resistencia antimicrobiana en aislamientos de Staphylococcus spp. obtenidos de leche bovina en Colombia. Rev Argent Microbiol. 1 de abril de 2020;52(2):121-30.

33. Zendejas-Manzo GS, Avalos-Flores $\mathrm{H}$, Soto-Padilla MY. Microbiología general de Staphylococcus aureus: Generalidades. Staphylococcus Aureus. 2014;25(3):15.

Conflicto de intereses: Ninguno declarado por los autores. Financiación: Ninguna declarada por los autores. Agradecimiento: Ninguno manifestado por los autores

\section{ACERCA DE LOS AUTORES}

Silvia Monserrath Torres Segarra. Bioquímica Farmacéutica, Universidad de Cuenca, Magister en Gerencia en Salud, Universidad Técnica Particular de Loja. Docente de Biotecnología, Universidad Politécnica Salesiana, Universidad Católica de Cuenca, Ecuador.

Karla Estefanía Pacheco Cárdenas. Química Farmaceuta, Universidad Católica de Cuenca. Máster en Bacteriología y Micología, Universidad de la Habana, Instituto de Medicina Tropical Pedro Kourí. Docente en la Carrera de Biofarmacia/ Bioquímica y Farmacia, Universidad Católica de Cuenca, Ecuador. 